\title{
Childhood growth and development and DNA methylation age in mid-life
}

\author{
Jane Maddock ${ }^{* *}$, Juan Castillo-Fernandez ${ }^{2}$, Andrew Wong ${ }^{1}$, George B. Ploubidis ${ }^{3}$, Diana Kuh', \\ Jordana T. Bell ${ }^{2}$ and Rebecca Hardy ${ }^{4}$
}

\begin{abstract}
Background: In the first study of its kind, we examine the association between growth and development in early life and DNAm age biomarkers in mid-life.

Methods: Participants were from the Medical Research Council National Survey of Health and Development ( $n=1376)$. Four DNAm age acceleration (AgeAccel) biomarkers were measured when participants were aged 53 years: AgeAccelHannum; AgeAccelHorvath; AgeAccelLevine; and AgeAccelGrim. Exposure variables included: relative weight gain (standardised residuals from models of current weight $z$-score on current height, and previous weight and height $z$-scores); and linear growth (standardised residuals from models of current height $z$-score on previous height and weight $z$-scores) during infancy ( $0-2$ years, weight gain only), early childhood ( $2-4$ years), middle childhood (4-7 years) and late childhood to adolescence (7-15 years); age at menarche; and pubertal stage for men at 14-15 years. The relationship between relative weight gain and linear growth and AgeAccel was investigated using conditional growth models. We replicated analyses from the late childhood to adolescence period and pubertal timing among 240 participants from The National Child and Development Study (NCDS).
\end{abstract}

Results: A 1SD increase in relative weight gain in late childhood to adolescence was associated with 0.50 years (95\% $\mathrm{Cl} 0.20,0.79$ ) higher AgeAccelGrim. Although the $\mathrm{Cl}$ includes the null, the estimate was similar in NCDS [0.57 years $(95 \% \mathrm{Cl}-0.01,1.16)]$ There was no strong evidence that relative weight gain and linear growth in childhood was associated with any other AgeAccel biomarker. There was no relationship between pubertal timing in men and AgeAccel biomarkers. Women who reached menarche $\geq 12$ years had 1.20 years $(95 \% \mathrm{Cl} 0.15,2.24)$ higher AgeAccelGrim on average than women who reached menarche $<12$ years; however, this was not replicated in NCDS and was not statistically significant after Bonferroni correction.

Conclusions: Our findings generally do not support an association between growth and AgeAccel biomarkers in mid-life. However, we found rapid weight gain during pubertal development, previously related to higher cardiovascular disease risk, to be associated with older AgeAccelGrim. Given this is an exploratory study, this finding requires replication.

Keywords: Epigenetic clock, Cohort, Longitudinal, Biological ageing, Biomarkers of ageing

\footnotetext{
*Correspondence: jane.maddock@ucl.ac.uk

${ }^{1}$ MRC Unit for Lifelong Health and Ageing at UCL, Faculty of Population Health, University College London, 1-19 Torrington Place, London WC1E $7 \mathrm{HB}, \mathrm{UK}$

Full list of author information is available at the end of the article
}

\begin{abstract}
Introduction
The demographic shift towards an ageing population is a recognised public health challenge. Despite increases in life expectancy, compression of morbidity is not evident and there is significant heterogeneity in the occurrence of age-related disease and functional capability among people of the same chronological age [1]. Definitions
\end{abstract}

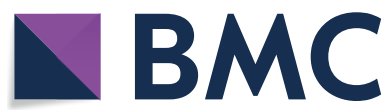

(c) The Author(s) 2021. Open Access This article is licensed under a Creative Commons Attribution 4.0 International License, which permits use, sharing, adaptation, distribution and reproduction in any medium or format, as long as you give appropriate credit to the original author(s) and the source, provide a link to the Creative Commons licence, and indicate if changes were made. The images or other third party material in this article are included in the article's Creative Commons licence, unless indicated otherwise in a credit line to the material. If material is not included in the article's Creative Commons licence and your intended use is not permitted by statutory regulation or exceeds the permitted use, you will need to obtain permission directly from the copyright holder. To view a copy of this licence, visit http://creativecommons.org/licenses/by/4.0/. The Creative Commons Public Domain Dedication waiver (http://creativeco mmons.org/publicdomain/zero/1.0/) applies to the data made available in this article, unless otherwise stated in a credit line to the data. 
of healthy ageing include survival to old age, delaying the onset of age-related diseases and maintaining function [2]. Ageing is a complex process involving changes at the molecular, cellular, physiological and functional level over time [3]. Biomarkers of ageing, which combine information from one or more of these processes, have been proposed as tools to capture healthy ageing [4]. A suitable biomarker of ageing should be better at predicting survival, onset of age-related disease and functional capability at later ages than chronological age alone.

Epigenetic mechanisms, specifically DNA methylation (DNAm), have been implicated in the ageing process [5]. Several DNAm-based biomarkers of ageing have been developed [6-11]. The first generation of these biomarkers used a data-driven elastic net regression method to identify specific DNAm sites (CpGs) that are highly predictive of chronological age. These DNAm-based biomarkers of ageing include the blood-based Hannum and the multi-tissue Horvath clocks [7, 8]. The second generation of DNAm-based biomarkers of ageing, the Levine clock (also referred to as PhenoAge) and GrimAge, use information about age-related traits and mortality in addition to chronological age [10]. The Levine clock was developed using composite age-related clinical physiological measures to identify associated CpGs from DNAm in whole blood [10, 11]. GrimAge was created by combining surrogate DNAm-based plasma protein estimates, DNAm smoking pack years estimates, chronological age and sex as a function of mortality [11]. It remains unclear exactly what aspects of ageing each of these biomarkers are capturing [12]. However, having a higher DNAm age independent of chronological age (denoted age acceleration, AgeAccel) for each of these biomarkers has been shown to be associated with an increased risk of premature all-cause mortality, cardiovascular disease and cancer with AgeAccelLevine and AgeAccelGrim showing stronger associations than AgeAccelHannum or AgeAccelHorvath [10, 11, 13-16]. We have also recently demonstrated that AgeAccelLevine and AgeAccelGrim are associated with markers of age-related physical and cognitive performance [17].

Using a life course approach can provide novel insights into how biological, behavioural and psychosocial processes over time affect healthy ageing [2]. Childhood is a sensitive period during which physiological changes can be initiated, leading to long-term health consequences in age-related physical and cognitive performance and age-related disease [18]. There may be a specific period during childhood where growth has a lasting impact on a particular age-related health outcome. Birth weight, child and adolescent weight and height gain have been associated with a range of age-related and disease outcomes, but patterns vary depending on the outcome [19-24].
Similarly, the timing of puberty has exhibited differential associations with later life heath; for example, younger age at puberty, particularly among women, is associated with higher risk of cardiovascular disease [25] and allcause mortality [26], but later puberty is associated with lower bone mineral density [27].

While a few studies in childhood and adolescence have examined how AgeAccelHorvath is associated with growth and pubertal timing (not vice versa) [28-30], to our knowledge, no study has examined how physical growth during childhood or pubertal timing is related to DNAm-based biomarkers of ageing in later life. In the largest of these previous studies $(n=1018)$, higher AgeAccelHorvath at birth was associated with higher average fat mass and faster growth in weight and BMI between birth and 17 years [30]. In a cross-sectional study of Finnish children aged $11-13$ years $(n=239)$, higher AgeAccelHorvath was associated with heavier weight-for-age, taller height-for-age and more advanced puberty based on Tanner Stage [29]. In a smaller longitudinal $(n=94)$ study of Chilean girls aged 9-13 years, higher AgeAccelHorvath was associated with earlier age at menarche [28]. These studies suggest that higher AgeAccelHorvath in early life is associated with more rapid growth and earlier development [28-30]. It is not known if this association is similar for other AgeAccel biomarkers, if it tracks across adulthood, or if growth and development in childhood has additional effects on AgeAccel that persist across the life course.

In this exploratory study using data from a subsample of a nationally representative British birth cohort, we investigate the impact of birth weight and physical growth during infancy (birth to 2 years), early childhood (2-4 years), middle childhood ( $4-7$ years) and late childhood to adolescence (7-15 years) and pubertal timing on four DNAm-based biomarkers of ageing in mid-life. We examine both linear growth and weight gain relative to linear growth to explore their potential separate effects [31]. Where possible we conducted a replication study among a sub-sample of participants from a British birth cohort born twelve years later.

\section{Methods}

\section{Participants}

Participants were from the Medical Research Council National Survey of Health and Development study (MRC NSHD, or 1946 British birth cohort). NSHD is one of the longest running birth cohorts worldwide, and participants have been followed up 24 times since birth. Details about this cohort have been published previously [32-34]. Briefly, the 5362 original NSHD participants were singleton births born in 1 week in March 1946 to married parents in England, Scotland or Wales. This 
study has been running for 75 years, and attrition has been documented in detail elsewhere [32]. When participants were 53 years contact was not attempted for the 2227 participants who had died, previously refused to take part, were living abroad or untraceable. While avoidable non-response in adulthood was highest among those with adverse socioeconomic circumstances and with low scores on childhood cognitive measures, study participants remain broadly representative of the native British population born in the early post-war years [3436]. Of the participants who were still alive and resident in England, Scotland or Wales when they were 53 years old, 3035 provided information during a home visit by a research nurse and blood samples were taken from those who consented. The data collection at age 53 years received multicentre research ethics committee approval, and informed consent was given by respondents to each set of questions and measures. A subset of participants at 53 years with blood samples who also had information on a wide range of health and age-related variables across the life course were selected for DNAm analyses $(n=1595)$. After quality control, 1376 participants with DNAm information who also had weight and/or height measured at least once between birth and 15 years were included in analyses.

\section{DNAm-based biomarkers of ageing}

DNAm from participant's blood samples was measured at $>850,000$ CpG sites using Infinium MethylationEPIC BeadChips and processed using the ENmix package [37] in $\mathrm{R}$ to obtain methylation beta-values for quality control purposes. In addition, signals with a detection $p$ value $>1 \times 10-6$ and a number of beads $<3$ were set to missing. Samples with missing data in $>5 \%$ of the CpGs were excluded. CpGs with missing data in $>5 \%$ of the samples were excluded. Samples identified with outlier values (more than three standard deviations from the mean or three interquartile ranges below the first or above the third quartiles) in bisulphite intensity, total intensity or beta-value distribution were excluded. Sample identity was verified by estimating the correlation $(r>0.90)$ between the 59 SNPs included in the methylation beadchips and imputed genotype data.

We used four DNAm-based biomarkers of ageing in this study: DNAm AgeHannum; DNAm AgeHorvath; DNAm AgeLevine; and DNAm GrimAge [7, 8, 10, 11]. There are 71, 353, 513 and 1030 CpG sites included in each clock, respectively. DNAm GrimAge and DNAm AgeLevine were developed using both the Infinium HumanMethylation450 BeadChip and the Infinium MethylationEPIC BeadChips, while DNAm AgeHannum and DNAm AgeHorvath used the Infinium HumanMethylation450 BeadChip only. Therefore, participants included in this study have all CpGs for DNAm GrimAge and DNAm AgeLevine and are missing 6 CpGs for DNAm AgeHannum and 19 CpGs for DNAm AgeHorvath. Previous studies have found that the DNAm age estimate is unaffected by platform differences [38]. Besides those CpG sites already expected to be missing, in this study few DNAm age CpG sites among a small number of participants did not pass quality control. Among all participants, the mean number of DNAm CpG sites that failed quality control was 0.40 (range 0-5) for DNAm AgeHorvath (excluding the $19 \mathrm{CpGs}$ that are not present in the EPIC array), 0.1 (range 0-2) for DNAm AgeHannum (excluding the $6 \mathrm{CpGs}$ that are not present in the EPIC array) and 2.46 (range 2-8) for DNAm Levine. Likewise, among DNAm CpG sites, the mean number of participants with a missing value was 1.70 (range 0-64) for DNAm AgeHorvath, 2.20 (range 0-58) for DNAm AgeHannum and 7.01 (range 0-1460) for DNAm Levine. Each DNAm-based biomarker was calculated using freely available software (https://labs.genet ics.ucla.edu/horvath/dnamage/) with the normalisation option and advanced analysis for blood samples. Input data were produced using ssNoob pre-processing of the DNA methylation arrays in minfi [39]. DNAm-based age biomarkers were estimated using the epigenetic age calculator which implements its own data processing and normalisation steps to minimise batch effects in methylome datasets obtained from different sources. Therefore, DNAm-based age biomarkers are robust to batch effects. Chronological age-independent DNAm-based biomarkers (residuals from a regression of DNAm age on chronological age) were calculated within this software to represent the difference between an individual's DNAm Age and chronological age: AgeAccelHannum, AgeAccelHorvath, AgeAccelLevine and AgeAccelGrim (in units of a year). Estimated blood cell counts (naïve and exhausted CD8+T-lymphocytes, CD4+T-lymphocytes, B cells, natural killer cells, monocytes and granulocytes) were also calculated within this software.

\section{Weight and height}

Weight $(\mathrm{kg})$ and height $(\mathrm{cm})$ in childhood were measured using standardised protocols at 2 (exact age not recorded), 4 (mean: 4.3 years; range $4.2-4.9$ years), 7 (mean: 7.1 years; range 6.92-7.75) and 15 years (mean: 14.54 years; range 14.33-15.17 years). Birth weight was extracted from birth records to the nearest quarter pound and converted to $\mathrm{kg}$. Birth length was not recorded. We conceptualised different periods of growth as: infancy (birth to 2 years); early childhood (2-4 years); middle childhood (4-7 years); and late childhood to adolescence (7-15 years which captures the full period of pubertal growth). All weight and height measures were 
converted to sex-specific $z$-scores using the mean and standard deviation to facilitate comparison of estimates between different ages.

\section{Pubertal timing}

Pubertal stage for boys at age 14-15 years was based on physical examination by school doctors of four criteria: visibility of pigmented pubic hair; the visibility of axillary hair; the development of genitalia; and whether the voice had broken [40]. Based on responses to these questions, boys were grouped into fully mature (i.e. those who experienced the earliest pubertal timing), advanced puberty, early puberty and pre-pubertal.

Age at menarche for girls was obtained from mother's reports when the girls were 14-15 years. Of those who had not reached menarche at this date $(n=188)$, age at menarche was obtained from self-report at 48 years $(n=94)$. A previous study found moderate agreement between prospective and retrospective measures of menarche [41].

Individual patterns of height and weight growth during puberty were estimated using the SITAR model of growth curve analysis and used in sensitivity analyses [42]. Briefly, the SITAR model summarises each individual's growth curve in terms of three parameters: size, tempo and velocity, each expressed relative to the mean curve. The tempo parameter indicates the relative timing of puberty based on the age at peak velocity with higher scores reflecting later pubertal timing.

\section{Additional variables}

We selected a number of additional variables a priori for descriptive purposes. Socioeconomic position (SEP) at 53 years (or 43 years if missing) was based on occupation grouped according to the Registrar General's social class and categorised into non-manual and manual. Similarly, father's SEP when the cohort member was 4 years old was categorised as non-manual or manual. Smoking status at 53 years was self-reported and categorised as current, ex-smoker or never smoker.

\section{Statistical analyses}

All analyses were conducted in Stata 14 using the four AgeAccel biomarkers as outcomes. Further mention of AgeAccel refers to all four biomarkers unless specified. Models using AgeAccel as an outcome were adjusted for sex and age (in months) when the DNA sample was taken.

We assessed if participants characteristics differed between those included in our main analyses and those who were not included in our analyses using $t$ tests and Chi-squared tests.
In preliminary analyses, we investigated associations between weight and height from infancy to adolescence and AgeAccel. The relation of weight and height $z$-scores with AgeAccel was examined using separate multiple regression models for each age for descriptive purposes. Since weight and height are correlated, these models were mutually adjusted for weight or height $z$-scores. Interactions between sex and height or weight $z$-scores were tested to assess whether associations were different for men and women.

For our main analyses, we used regression with conditional growth measures [43-46]. This method involves computing a sex-specific conditional growth measure, i.e. the standardised residuals from a regression of current size on the previous size measure. These conditional growth variables are by definition uncorrelated with size at the previous age and represent the deviation of a participant's current size from that expected given their previous measure and the growth of the other participants in the sample. In order to examine the effects of both linear growth and relative weight gain, we used an approach described by Adair and colleagues [31]. Conditional relative weight was calculated as the standardised residuals of current weight $z$-score accounting for previous weight and height $z$-score as well as current height $z$-score. Conditional height was calculated as the standardised residuals from a regression of current height $z$-score on previous height and weight $z$-scores (but not current weight $z$-score). For each period of childhood, linear regression models were used to examine the association between the conditional growth measure and AgeAccel.

Linear regression models were also used to examine the association between pubertal timing and AgeAccel. For girls, we used both continuous age at menarche and a dichotomised score of $<12$ years and $\geq 12$ years to examine early menarche. For boys, pubertal stage at 14-15 years was assessed.

We conducted 44 tests in our main analyses. We apply a Bonferroni correction to account for multiple testing.

\section{Sensitivity analyses}

We conducted five main sensitivity analyses. To account for the exact age at height and weight measurement in months, we repeated the growth analyses using sexspecific standard deviation scores for weight and heights using internally generated growth charts known as the LMS method ( $\mathrm{L}=$ skewness; $\mathrm{M}=$ median; $\mathrm{S}=$ coefficient of variation) $[47,48]$. Using an internally generated growth chart is preferable to an external reference for historical cohorts [49]. Second, while our primary aim in this study was to investigate associations between conditional growth during early life and AgeAccel, irrespective of adult body size, we conduct sensitivity analyses 
adjusting for body size at 53 years to assess whether any observed associations with growth are mediated through body size in adulthood. Third, since more participants had information on the SITAR variables than timing of puberty, and SITAR can be an alternative approach to capture pubertal timing, we repeated the analysis using height-tempo. Fourth, we adjusted the conditional growth models and timing of puberty for estimated cell counts (naïve and exhausted CD8 + T-lymphocytes, CD4+T-lymphocytes, B cells, natural killer cells, monocytes and granulocytes). Finally, a subsample of participants in NSHD also had AgeAccel measures at 60-64 years $(n=482)$. Therefore, we investigated if the associations between growth and development in early life and AgeAccel were consistent when AgeAccel was measured approximately ten years later.

\section{Replication}

We used data from the National Child and Development Study (NCDS; 1958 British birth cohort) to replicate findings where possible. NCDS is a nationally representative birth cohort where participants $(n=18,558)$ were born within the same week in 1958 and have been followed up 11 times to date. Details of this cohort along with attrition have been described in detail previously [50]. Briefly, by 44 years 2555 cohort members had died or emigrated and 9377 (58.6\% of the eligible sample) participated in a biomedical survey. Of these, 3641 participants had data from every sweep and gave consent for DNA extraction. For this analysis, we used data from a small subsample of these NCDS participants $(n=240)$. These participants were randomly selected from those that had no missing data on key health and demographic variables. When comparing these 240 participants to the remaining participants at 45 years, there were no differences in weight, height or age at menarche. However, men included in our analyses were less likely to experience puberty at a later stage than those not included (data available on request). AgeAccel measures were obtained using the same methods as NSHD. Height and weight were measured at ages 7,11 and 16 years. We used weight $z$-scores in NCDS at 7 and 16 years to replicate analyses for growth during the late childhood to adolescence period and AgeAccel observed in NSHD using regression with conditional growth measures as outlined above.

Pubertal stage among boys in NCDS was assessed by a physical examination by trained medical personnel when participants were 16 years. We used the following criteria to assign boys as fully mature (i.e. those who experienced the earliest pubertal timing) or later puberty for comparison with NSHD: visibility of pigmented pubic hair; the visibility of axillary hair; whether the voice had broken; and the visibility of facial hair. Age at menarche was reported by the medical officer (or parent if missing) at the 16 year examination. Two women in the NCDS sample had not reached menarche by 16 years and were coded as greater than 16 years.

\section{Results}

Descriptive characteristics of participants included in the main analysis $(n=1376)$ are outlined in Table 1 . There were no major differences in body size, pubertal timing, smoking status or SEP among participants included in our main analysis versus all other NSHD participants responding to the 53 year data collection $(n=1659$, Additional file 1: Table 1).

The median absolute difference between DNAm AgeHannum, DNAm AgeHorvath, DNAm Levine and DNAm GrimAge and chronological age at 53 years was 11.1, 4.0, 14.7 and 2.8 years, respectively. The correlation coefficients between the different AgeAccel biomarkers ranged from $r=0.1$ for AgeAccelHorvath and AgeAccelGrim to $r=0.4$ for AgeAccelLevine with AgeAccelHannum, AgeAccelHorvath and AgeAccelGrim (Additional file 1: Table 2).

\section{Preliminary analyses: AgeAccel at 53 years and weight and height $\mathrm{z}$-scores at each time point in childhood}

We find little evidence of any strong relationships between weight and height at each age separately with any AgeAccel biomarker at 53 years (Fig. 1). The largest differences in coefficients from one age to the next, which are indicative of the importance of change in size, are observed between 7 and 15 years for weight in relation to AgeAccelGrim and also with AgeAccelHannum and AgeAccelLevine. There were also differences in coefficients observed between 2 and 4 years and 4 and 7 years for height in relation to AgeAccelHanum and AgeAccelLevine. There was no consistent evidence for sex differences except for AgeAccelHorvath where there was evidence for an interaction between sex and birth weight $z$-score and weight $z$-score at 4 years $\left(p_{\text {interaction }} \leq 0.03\right.$; 0.39 [95\% CI 0.05-0.73] for men and -0.11 [95\% CI -0.41 to 0.18$]$ for women).

\section{Main analyses: AgeAccel at 53 years and conditional relative weight and linear growth in childhood}

There was no evidence that relative weight gain and linear growth during childhood was associated with AgeAccelHannum or AgeAccelHorvath (Table 2).

In the conditional growth models (Table 2), a 1 SD increase in relative weight gain between the ages of 7 and 15 years was associated with 0.50 years $(95 \%$ CI 0.20 , 0.79) higher AgeAccelGrim. This association remained statistically significant after Bonferroni correction. 
Table 1 Descriptive characteristics $(n=1376)$

\begin{tabular}{|c|c|c|c|c|}
\hline & \multicolumn{2}{|c|}{ Men } & \multicolumn{2}{|c|}{ Women } \\
\hline & $N$ & Mean (SD) & $N$ & Mean (SD) \\
\hline \multicolumn{5}{|l|}{ AgeAccel measures at 53 years } \\
\hline DNAm age Hannum (years) & 656 & $43.1(4.3)$ & 720 & \\
\hline AgeAccelHannum & 656 & $0.79(4.28)$ & 720 & $-0.66(3.95)$ \\
\hline DNAm age Horvath (years) & 656 & $50.7(4.2)$ & 720 & $49.6(3.9)$ \\
\hline AgeAccelHorvath & 656 & $0.54(4.15)$ & 720 & $-0.54(3.86)$ \\
\hline DNAm age Levine (years) & 656 & $39.0(5.6)$ & 720 & $38.9(5.6)$ \\
\hline AgeAccelLevine & 656 & $0.06(5.59)$ & 720 & $-0.02(5.61)$ \\
\hline GrimAge (years) & 656 & $58.0(5.1)$ & 720 & $55.3(4.8)$ \\
\hline AgeAccelGrim & 656 & $1.40(5.14)$ & 720 & $-1.28(4.79)$ \\
\hline \multicolumn{5}{|l|}{ Weight (kg) } \\
\hline At birth & 656 & $3.46(0.53)$ & 719 & $3.34(0.49)$ \\
\hline At 2 years & 586 & $13.23(1.50)$ & 617 & $12.54(1.39)$ \\
\hline At 4 years & 626 & $17.42(2.10)$ & 672 & $16.84(2.02)$ \\
\hline At 7 years & 656 & $23.00(2.84)$ & 643 & $22.39(3.11)$ \\
\hline At 15 years & 624 & $51.83(9.73)$ & 608 & $51.53(8.58)$ \\
\hline \multicolumn{5}{|l|}{ Height (cm) } \\
\hline At 2 years & 576 & $86.11(5.08)$ & 598 & $84.81(4.44)$ \\
\hline At 4 years & 611 & $103.47(5.15)$ & 653 & $102.54(4.87)$ \\
\hline At 7 years & 656 & $120.49(5.86)$ & 678 & $119.42(5.36)$ \\
\hline At 15 years & 623 & $162.24(9.17)$ & 611 & $158.51(6.05)$ \\
\hline \multirow[t]{2}{*}{ Age at menarche (years) } & & & 617 & $13.11(1.26)$ \\
\hline & & $N$ & & $\%(N)$ \\
\hline \multicolumn{5}{|c|}{ Pubertal stage at 14-15 years (men) } \\
\hline Fully mature* & & 172 & & $26.22(172)$ \\
\hline Advanced puberty & & 211 & & $32.16(211)$ \\
\hline Early puberty & & 200 & & $30.49(200)$ \\
\hline Pre-pubertal & & 73 & & $11.13(73)$ \\
\hline
\end{tabular}

AgeAccel: Age Acceleration

*Fully mature group are the group who experienced the earliest pubertal timing

For linear growth, there was modest evidence that more rapid growth between 2 and 4 years was associated with lower AgeAccelLevine $(-0.39$ years $[95 \%$ CI $-0.74,-0.04])$. However, this association was not statistically significant after Bonferroni correction.

\section{Main analyses: AgeAccel at $\mathbf{5 3}$ years and pubertal timing}

There was no relationship between pubertal timing in men and any of the AgeAccel biomarkers at 53 years (Table 3). Women who reached menarche at 12 years or older had 1.20 years $(95 \%$ CI $0.15,2.24)$ higher AgeAccelGrim on average than women who reached menarche younger than 12 years. This association was not statistically significant after Bonferroni correction.

\section{Sensitivity analyses}

We observed no substantial differences in results after applying age-adjusted standard deviation scores for weight and heights using internally generated growth charts (Additional file 1: Table 3).

Adjusting for body size at 53 years did not attenuate the association between relative weight gain between the ages of 7 and 15 years and AgeAccelGrim (0.57 [95\% CI $0.25,0.90])$.

We observed no associations between the SITAR measure of pubertal timing in men or women with any AgeAccel biomarker (Additional file 1: Table 4).

Adjusting the conditional growth models for estimated cell composition attenuated the estimates; the estimate for the association of linear growth between 2 


\section{DNAm Age Acceleration at 53 years and weight z-scores}
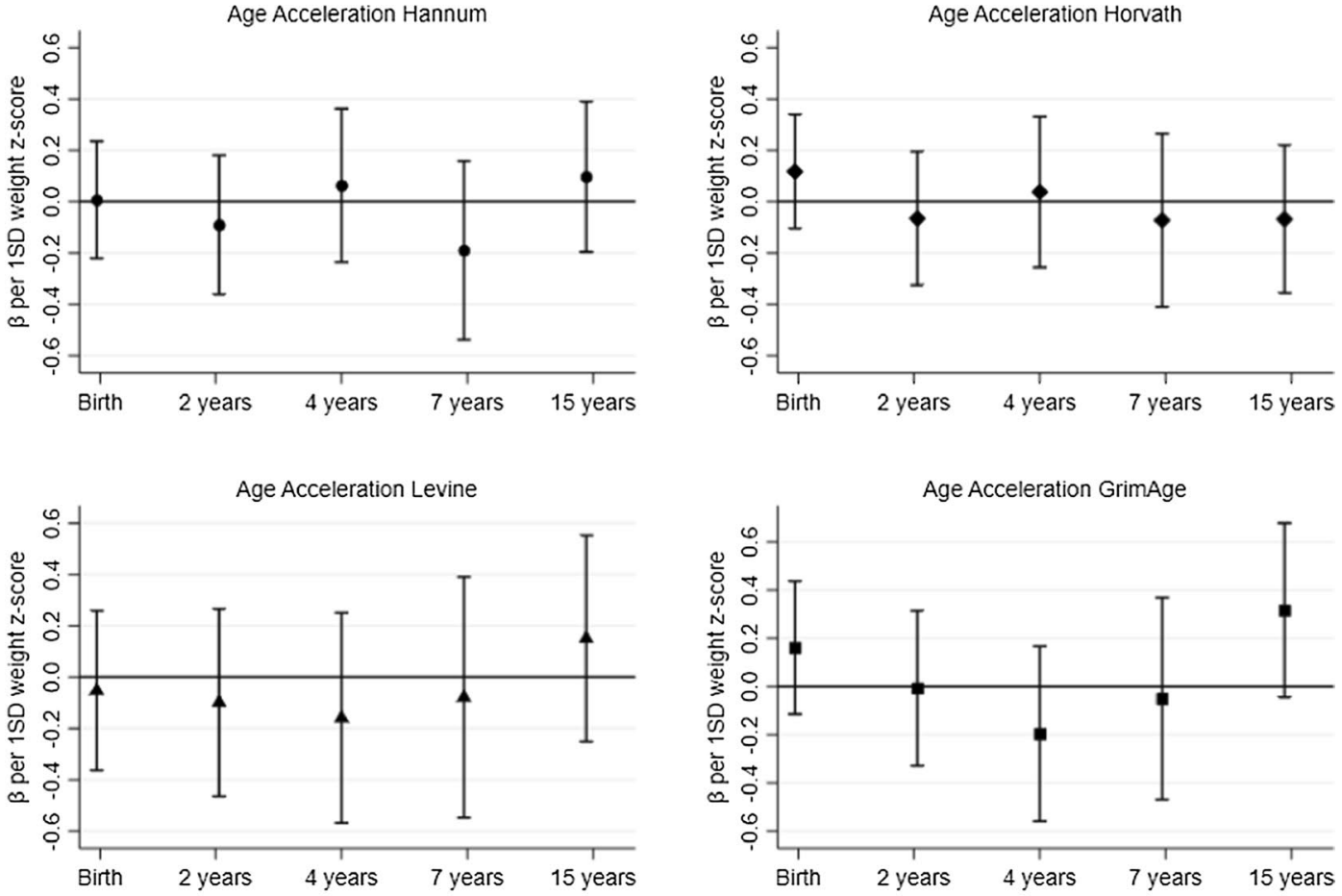

DNAm Age Acceleration at 53 years and height z-scores
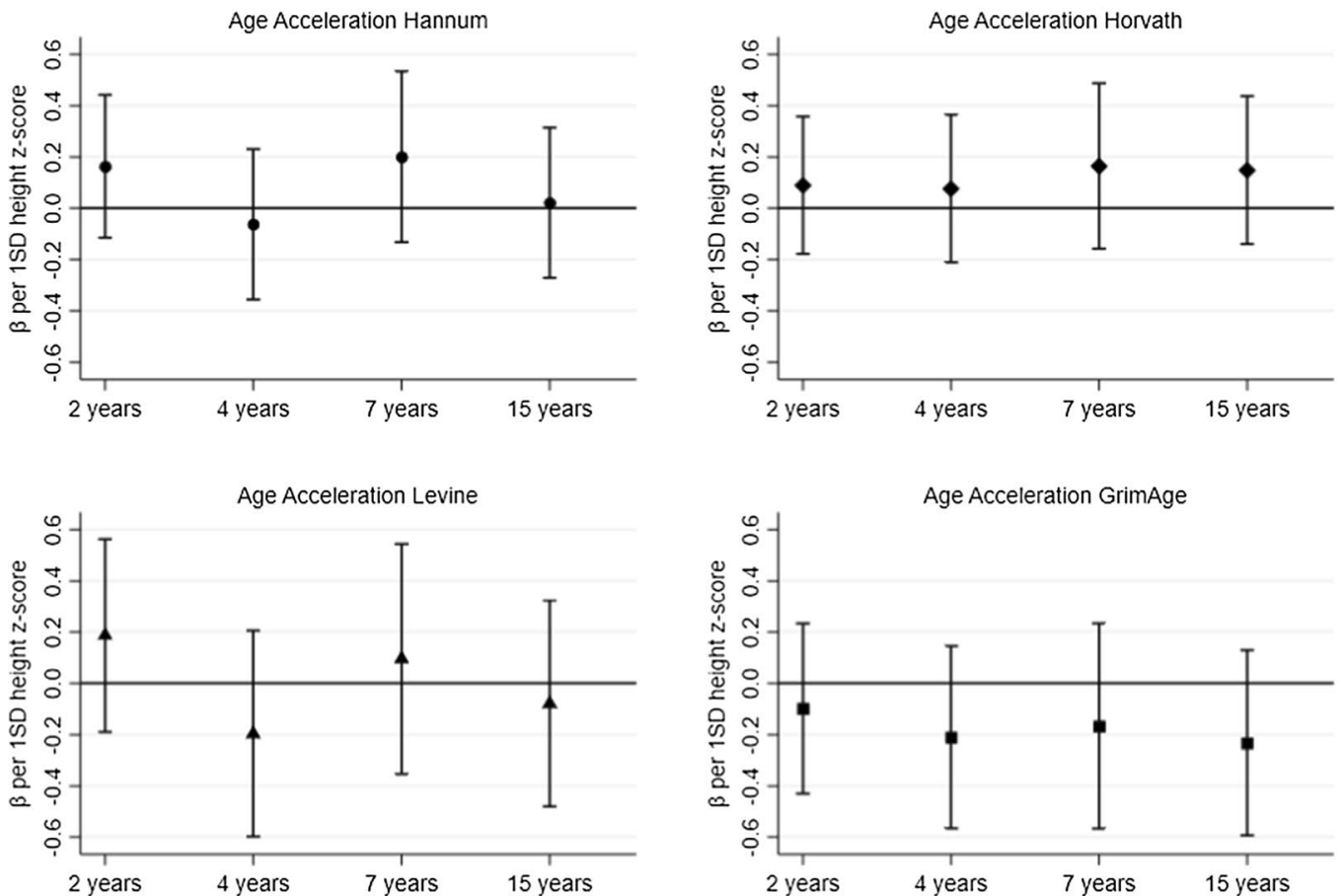

Fig. 1. Preliminary results for DNAm Age Acceleration at 53 years and weight and height z-scores. Adjusted for height/weight z-score, age in months at 53 years and sex. Each coefficient represents mean change in AgeAccel (y) for a 1 SD increase in height/weight. Separate analyses were conducted at each age 
Table 2 DNAm age acceleration at 53 years and conditional growth

\begin{tabular}{|c|c|c|c|c|c|c|c|c|c|}
\hline & \multirow[t]{2}{*}{$N$} & \multicolumn{2}{|l|}{ AgeAccelHannum } & \multicolumn{2}{|l|}{ AgeAccelHorvath } & \multicolumn{2}{|l|}{ AgeAccelLevine } & \multicolumn{2}{|l|}{ AgeAccelGrim } \\
\hline & & $\begin{array}{l}\text { Coefficient (95\% } \\
\text { Cl) }\end{array}$ & $P$ value & $\begin{array}{l}\text { Coefficient (95\% } \\
\mathrm{Cl})\end{array}$ & $P$ valve & $\begin{array}{l}\text { Coefficient (95\% } \\
\text { Cl) }\end{array}$ & $P$ value & $\begin{array}{l}\text { Coefficient (95\% } \\
\text { Cl) }\end{array}$ & $P$ value \\
\hline \multicolumn{10}{|l|}{ Relative weight gain } \\
\hline $\begin{array}{l}\text { RWG between } \\
\text { birth and } 2 \text { years }\end{array}$ & 1127 & $-0.09(-0.34,0.16)$ & 0.48 & $-0.09(-0.34,0.15)$ & 0.44 & $-0.05(-0.39,0.29)$ & 0.76 & $-0.08(-0.38,0.22)$ & 0.62 \\
\hline $\begin{array}{l}\text { RWG between } 2 \\
\text { and } 4 \text { years }\end{array}$ & 1065 & $0.11(-0.15,0.37)$ & 0.41 & $0.18(-0.07,0.43)$ & 0.16 & $-0.07(-0.42,0.28)$ & ) 0.69 & $-0.11(-0.42,0.19)$ & 0.47 \\
\hline $\begin{array}{l}\text { RWG between } 4 \\
\text { and } 7 \text { years }\end{array}$ & 1168 & $-0.17(-0.43,0.08)$ & 0.18 & $-0.08(-0.33,0.16)$ & 0.51 & $0.05(-0.29,0.40)$ & ) 0.76 & $0.08(-0.23,0.39)$ & 0.60 \\
\hline $\begin{array}{l}\text { RWG between } 7 \\
\text { and } 15 \text { years }\end{array}$ & 1161 & $0.16(-0.08,0.40)$ & 0.19 & $-0.01(-0.24,0.23)$ & 0.94 & $0.22(-0.11,0.54)$ & ) 0.19 & $0.50(0.20,0.79)$ & $<0.001^{*}$ \\
\hline \multicolumn{10}{|l|}{ Linear growth } \\
\hline $\begin{array}{l}\text { CLG between } 2 \\
\text { and } 4 \text { years }\end{array}$ & 1085 & $-0.06(-0.31,0.20)$ & 0.67 & $0.07(-0.18,0.32)$ & 0.57 & $\begin{array}{l}-0.39(-0.74 \\
-0.04)\end{array}$ & $0.03^{* *}$ & $-0.24(-0.55,0.06)$ & 0.12 \\
\hline $\begin{array}{c}\text { CLG between } 4 \\
\text { and } 7 \text { years }\end{array}$ & 1204 & $0.11(-0.12,0.33)$ & 0.36 & $-0.04(-0.26,0.19)$ & 0.75 & $0.16(-0.15,0.47)$ & 0.32 & $0.01(-0.27,0.28)$ & 0.97 \\
\hline $\begin{array}{l}\text { CLG between } 7 \\
\text { and } 15 \text { years }\end{array}$ & 1174 & $0.11(-0.13,0.35)$ & 0.36 & $0.03(-0.20,0.26)$ & 0.78 & $0.01(-0.31,0.33)$ & 0.97 & $0.14(-0.15,0.43)$ & 0.35 \\
\hline
\end{tabular}

Adjusted for age in months at 53 years and sex. RWG: Relative weight gain, i.e. standardised residuals from regression of present weight $z$-score on previous weight and height $z$-scores and present height $z$-score

CLG: Conditional linear growth, i.e. standardised residuals from regression of present height $z$-score on previous height and weight $z$-scores

${ }^{*} p=0.04$ after Bonferroni correction

${ }^{* *} p=1.0$ after Bonferroni correction

Table 3 DNAm age acceleration at 53 years and pubertal timing

\begin{tabular}{|c|c|c|c|c|c|c|c|c|c|}
\hline & \multirow[t]{2}{*}{$N$} & \multicolumn{2}{|l|}{ AgeAccelHannum } & \multicolumn{2}{|l|}{ AgeAccelHorvath } & \multicolumn{2}{|l|}{ AgeAccelLevine } & \multicolumn{2}{|l|}{ AgeAccelGrim } \\
\hline & & $\begin{array}{l}\text { Coefficient (95\% } \\
\text { Cl) }\end{array}$ & $P$ value & $\begin{array}{l}\text { Coefficient (95\% } \\
\mathrm{Cl})\end{array}$ & $P$ value & $\begin{array}{l}\text { Coefficient ( } 95 \% \\
\text { Cl) }\end{array}$ & $P$ value & $\begin{array}{l}\text { Coefficient (95\% } \\
\text { Cl) }\end{array}$ & $P$ value \\
\hline \multicolumn{10}{|l|}{ Women } \\
\hline $\begin{array}{l}\text { Age at menarche } \\
\text { (years) }\end{array}$ & 617 & $0.07(-0.18,0.32)$ & 0.59 & $0.04(-0.20,0.29)$ & 0.73 & $\begin{array}{l}-0.02(-0.37 \\
0.33)\end{array}$ & 0.91 & $0.22(-0.08,0.53)$ & 0.15 \\
\hline \multicolumn{10}{|l|}{ Age at menarche } \\
\hline$<12$ years & 96 & Ref & & Ref & & Ref & & Ref & \\
\hline$\geq 12$ years & 521 & $0.37(-0.49,1.23)$ & 0.40 & $0.27(-0.57,1.11)$ & 0.53 & $0.73(-0.48,1.94)$ & 0.24 & $1.20(0.15,2.24)$ & $0.03^{* * *}$ \\
\hline \multicolumn{10}{|c|}{ Men: Pubertal status at $14-15$ years } \\
\hline Fully mature* & 172 & Ref & & Ref & & Ref & & Ref & \\
\hline Advanced puberty & 211 & $0.17(-0.70,1.03)$ & $0.56^{* *}$ & $\begin{array}{l}-0.36(-1.20 \\
0.48)\end{array}$ & $0.83^{* *}$ & $0.95(-0.17,2.08)$ & $0.08^{* *}$ & $\begin{array}{l}-0.29(-1.33 \\
0.75)\end{array}$ & $0.93^{* *}$ \\
\hline Early puberty & 200 & $0.39(-0.48,1.27)$ & & $\begin{array}{l}-0.18(-1.03 \\
0.66)\end{array}$ & & $0.50(-0.64,1.64)$ & & $\begin{array}{l}-0.05(-1.10 \\
1.00)\end{array}$ & \\
\hline Pre-pubertal & 73 & $\begin{array}{l}-0.40(-1.57 \\
0.77)\end{array}$ & & $\begin{array}{l}-0.39(-1.54 \\
0.75)\end{array}$ & & $\begin{array}{l}-0.83(-2.36 \\
0.70)\end{array}$ & & $\begin{array}{l}-0.30(-1.71 \\
1.11)\end{array}$ & \\
\hline Fully mature & 172 & Ref & & Ref & & Ref & & Ref & \\
\hline Later puberty & 484 & $0.18(-0.57,0.92)$ & 0.64 & $\begin{array}{l}-0.29(-1.02 \\
0.43)\end{array}$ & 0.43 & $0.50(-0.48,1.47)$ & 0.32 & $\begin{array}{l}-0.19(-1.09 \\
0.70)\end{array}$ & 0.67 \\
\hline
\end{tabular}

Adjusted for age in months at 53 years. *Fully mature group are the group who experienced the earliest pubertal timing

**P value from latest comparing models with and without categorical puberty variable

${ }^{* * *} P=1.0$ after Bonferroni correction 
and 4 years and AgeAccelLevine was halved to -0.20 (95\% CI -0.50 to 0.10 ) (Additional file 1: Table 5). The association between age at menarche and AgeAccelGrim was greatly attenuated to 0.53 years $(95 \% \mathrm{CI}-0.46,1.53)$ when adjusting for estimated cell composition (Additional file 1: Table 6).

When using AgeAccel at 60-64 years as the outcome where the sample size is smaller, the association between relative weight gain between 7 and 15 years and AgeAccelGrim was weaker than that observed at 53 years (0.38 [95\% CI -0.04 to 0.79] (Additional file 1: Table 7). An association between relative weight gain between 7 and 15 years and AgeAccelLevine was observed at 60-64 years $(0.69$ [95\% CI $0.12,1.26]$. The estimated association between linear growth between 2 and 4 years and AgeAccelLevine was similar $(-0.37$ [95\% CI -0.97 to 0.22$]$ ) to that found at age 53 years.

The direction of the estimates between pubertal timing and AgeAccel using the subsample from NSHD at 60-64 years was in a similar direction as 53 years. Associations between age at menarche ( $\geq 12$ years versus $<12$ years) and AgeAccelLevine at 60-64 years were stronger compared with AgeAccel biomarkers from 53 years (Additional file 1: Table 8). Among men, much larger estimates were observed when compared with 53 years. For example, compared to men with early puberty, those with later puberty had 1.27 years lower (95\% CI - 2.47, - 0.06) AgeAccelGrim at 60-64 years and 0.19 years lower $(95 \% \mathrm{CI}-1.09,0.70)$ at 53 years.

\section{Replication in NCDS}

A 1 SD increase in relative weight gain between the ages of 7 and 16 years was associated with 0.57 (95\% CI -0.01 to 1.16) years higher AgeAccelGrim (Table 4). Although this association crossed the null, the estimate is similar to that observed in NSHD (0.50 95\% CI 0.20-0.79). There was no association between pubertal timing and any AgeAccel biomarker in NCDS.

\section{Discussion}

We did not find strong evidence of associations between growth in early life and AgeAccel biomarkers in midadulthood. We did observe an association between faster weight gain during pubertal growth and higher AgeAccelGrim in mid-adulthood in NSHD. A similar estimate of the association was observed in the smaller sample from the NCDS, although the CI included the null value.

There are no previous studies with which to directly compare our findings. However in an English study, higher birth weight was correlated with higher AgeAccelHorvath at 7 years but with lower AgeAccelHorvath by 17 years, with no information for correlations beyond

Table 4 DNAm age acceleration at 45 years, conditional growth between 7 and 16 years and pubertal timing in the National Child and Development Study

\begin{tabular}{|c|c|c|c|c|c|c|c|c|c|}
\hline & \multirow[t]{2}{*}{$N$} & \multicolumn{2}{|l|}{ AgeAccelHannum } & \multicolumn{2}{|l|}{ AgeAccelHorvath } & \multicolumn{2}{|l|}{ AgeAccelLevine } & \multicolumn{2}{|l|}{ AgeAccelGrim } \\
\hline & & $\begin{array}{l}\text { Coefficient }(95 \% \\
\mathrm{CI})\end{array}$ & $P$ value & $\begin{array}{l}\text { Coefficient }(95 \% \\
\mathrm{CI})\end{array}$ & $P$ valve & $\begin{array}{l}\text { Coefficient }(95 \% \\
\mathrm{CI})\end{array}$ & $P$ value & $\begin{array}{l}\text { Coefficient }(95 \% \\
\text { CI) }\end{array}$ & $P$ value \\
\hline \multicolumn{10}{|l|}{ Conditional growth } \\
\hline $\begin{array}{l}\text { RWG between } 7 \\
\text { and } 16 \text { years }\end{array}$ & 240 & $\begin{array}{l}-0.04(-0.48 \\
0.39)\end{array}$ & 0.84 & $0.23(-0.25,0.70)$ & 0.35 & $0.20(-0.47,0.86)$ & 0.56 & $0.57(-0.01,1.16)$ & 0.06 \\
\hline \multicolumn{10}{|l|}{ Pubertal timing } \\
\hline \multicolumn{10}{|l|}{ Women } \\
\hline $\begin{array}{l}\text { Age at menarche } \\
\text { (years) }^{*}\end{array}$ & 112 & $\begin{array}{c}-0.21(-0.76 \\
0.33)\end{array}$ & 0.44 & $\begin{array}{c}-0.20(-0.77 \\
0.38)\end{array}$ & 0.50 & $\begin{array}{c}-0.21(-0.94 \\
0.53)\end{array}$ & 0.58 & $\begin{array}{c}-0.08(-0.71 \\
0.55)\end{array}$ & 0.80 \\
\hline \multicolumn{10}{|l|}{ Age at menarche } \\
\hline$<12$ years & 12 & Ref & & Ref & & Ref & & Ref & \\
\hline$\geq 12$ years & 100 & $\begin{array}{l}-0.77(-3.02 \\
1.47)\end{array}$ & 0.50 & $\begin{array}{c}-0.71(-3.07 \\
1.65)\end{array}$ & 0.55 & $\begin{array}{l}-1.06(-4.07 \\
1.96)\end{array}$ & 0.49 & $\begin{array}{l}-0.01(-2.61 \\
2.58)\end{array}$ & 0.99 \\
\hline \multicolumn{10}{|l|}{$\begin{array}{l}\text { Men: Pubertal sta- } \\
\text { tus at } 16 \text { years }\end{array}$} \\
\hline Fully mature ${ }^{* *}$ & 13 & Ref & & Ref & & Ref & & Ref & \\
\hline Later puberty & 99 & $0.08(-1.77,1.93)$ & 0.93 & $\begin{array}{l}-0.002(-2.050 \\
2.047)\end{array}$ & 1.00 & $\begin{array}{l}-0.99(-4.29 \\
2.31)\end{array}$ & 0.55 & $\begin{array}{l}-1.50(-4.38 \\
1.39)\end{array}$ & 0.31 \\
\hline
\end{tabular}

Adjusted for sex. RWG: Relative weight gain, i.e. standardised residuals from regression of weight $z$-score at 16 years on weight and height $z$-scores at 7 years and height $z$-score at 16 years. CLG: Conditional linear growth, i.e. standardised residuals from regression of height $z$-score at 16 years on height and weight $z$-scores at 7 years

*Those starting after 16 years coded as $\geq 16$ years

**Fully mature group are the group who experienced the earliest pubertal timing 
adolescence [51]. In the same study looking at the association in the other direction, higher AgeAccelHorvath at birth was associated with more rapid childhood and adolescent development including faster weight and BMI gains between childhood and adolescence [30]. Similarly, in a cross-sectional study of Finnish children aged 11-13 years $(n=239)$, higher AgeAccelHorvath was associated with heavier weight-for-age and taller heightfor-age [29]. We did not observe any strong associations with birth weight and AgeAccelHorvath in mid-life, suggesting that if these associations do exist, they may not persist into adulthood.

We did observe a relationship between faster gains in weight during pubertal growth with AgeAccelGrim. Among adults, there is a consistent association between higher BMI and higher AgeAccel in all four biomarkers $[10,11,52]$. Since rapid pubertal weight gain is associated with higher adult BMI, our findings suggest that the relationship we observed with AgeAccelGrim in mid-life may have been at least partly established in early life. In sensitivity analyses, we found that adjusting for adult BMI did not attenuate the association between faster gains in weight during pubertal growth and AgeAccelGrim, suggesting that the association is not fully mediated by body size in adulthood.

Our finding of an association between faster linear growth in early childhood and higher AgeAccelLevine among NSHD participants should be interpreted with caution. The estimates we observed for the association between faster linear growth in early childhood and higher AgeAccelLevine were not as large as the estimates for rapid weight gain in adolescence and did not remain statistically significant after adjusting for multiple testing. Adjusting for cell composition attenuated the association observed between linear growth and AgeAccelLevine however, since cell composition is a component in the creation of DNAm age Levine, this could be an over adjustment. We were unable to replicate this finding due to a lack of early life growth measures in NCDS.

While two previous studies in Finland and Chile observed that higher AgeAccelHorvath among children aged 9-13 years was associated with more advanced puberty based on Tanner stage or earlier age at menarche $[28,29]$, to our knowledge no previous study has examined the relationship between pubertal timing and AgeAccel biomarkers in later life. We found no evidence for an association between pubertal timing in men and any of the AgeAccel biomarkers at 53 years in NSHD or at 44 years in NCDS. Early puberty was associated lower AgeAccelGrim at 60-64 years among men in NSHD; however, this was a small sample and would need replication in a larger study. An association between older age at menarche and higher AgeAccel was observed in
NSHD at both 53 years and $60-64$ years. This finding is unexpected as older age at menarche is generally associated with better age-related outcomes and reduced risk of mortality [26]. This association did not remain statistically significant after adjusting for multiple testing, was not replicated in NCDS or when using age at menarche as a continuous variable. We also observed no association when SITAR variables were used to represent pubertal timing. Previous analysis in NSHD found no association between age at menarche and all-cause mortality, suggesting that different mortality rates by pubertal timing in women are unlikely to have biased this finding [53]. In sensitivity analyses, we found that the associations between older age at menarche and higher AgeAccel attenuated following adjustment for cell composition. As there is some evidence that pubertal timing is associated with white blood cell counts [54], it is possible that blood cell counts confounded our observed association. With all this in mind, these associations should be interpreted with caution and require further investigation in larger samples.

Our main results are based on relatively young participants at 53 years. While age-related disease may not always be evident at this age, it is possible that age-related DNAm changes are occurring. In order to examine if our observed associations change with age we repeated analyses on a subsample of NSHD participants who also had AgeAccel measures ten years later. We found that the estimates at 60-64 years were generally in the same direction but that some associations were weaker and some were stronger at 60-64 years. As participants age and accumulate more age-related changes the effect of early life development may become more evident. However, given the small sample size at 60-64 years, these findings would need replication in larger studies.

The use of DNAm to predict biological age is a newly emerging field, and there are many unknowns. We observed a large difference between the DNAm age biomarkers and chronological age. For example, DNAm age Levine was 14.7 years younger than chronological age in NSHD and 6.7 years younger in NCDS. This may indicate that participants in our analyses were unusually healthy; however, we do not observe this when comparing the analytical sample to the full cohort (Additional file 1: Table 1). This underestimation of chronological age has been observed in previous studies of the original DNAm age measures in healthy older population samples [55] and may partly reflect tissue-specific calibration issues in samples obtained from mid-life and at older age [56]. It is difficult to ascertain what aspects of ageing these biomarkers are capturing and the underlying biology of these relationships [12]. For this reason, we examined four AgeAccel biomarkers with the aim 
of informing future studies. We observed weak correlations between the four AgeAccel biomarkers, suggesting that they are not necessarily capturing the same underlying ageing construct. There are a few differences between how these biomarkers were constructed that may explain the differences in the results. The first generation of biomarkers are generally considered chronological age predictors, and the models were trained (i.e. the $\mathrm{CpG}$ sites and weights were determined) primarily using cross-sectional data. The second-generation biomarkers used additional age-related outcomes to select the CpG sites and were trained using longitudinal data [12]. The nature of the relationships in our study may provide more insight into the utility of these biomarkers. Assuming that growth in early life has an effect on age-related health outcomes, our finding of no associations between growth from infancy to middle childhood in any of the AgeAccel biomarkers suggests that these biomarkers are not fully capturing the effects of early life growth on agerelated conditions. However, the finding of an association between faster gains in weight during adolescence and higher AgeAccelGrim is similar to that seen with cardiovascular risk factors such as obesity, blood pressure and vascular structure and cardiovascular disease [19-21, 57]. This may suggest that the second-generation AgeAccel biomarkers (particularly AgeAccelGrim) is capturing a cardiovascular pathway, perhaps due to the inclusion of cardiovascular risk-factors in their construction.

The main strengths of this study are the inclusion of participants from two well characterised prospective population-based birth cohorts, the prospective repeated measures of body size from infancy, and the replication of the main finding. There are also a number of limitations to keep in mind when interpreting these findings. As with all prospective cohort studies, there is attrition in NSHD; however, at 53 years respondents were found to be generally representative of the white British population [32]. We observed no major differences in sociodemographic characteristics between participants included in our analyses and those who responded to the 53 year data collection but were not included in our analyses. Our replication cohort consisted of a small subsample of NCDS. While these participants share similar attributes to the full NCDS sample, they are not representative and findings from our study would need to be replicated among study samples that are representative of the target population. For both cohorts, there is a possibility that collider bias could have been introduced, as the selection for having DNAm was from those with complete growth and development data in childhood. If having lower DNAm age and faster weight gain was associated with participation, the estimates may have been biased [58]. Where possible we repeated our analyses in a subsample from NCDS to replicate findings. Since measures were not obtained from exactly the same ages we were unable to test for replication of the associations in infancy, early childhood or middle childhood. Similarly, timing of puberty was assessed slightly differently between the studies which may have accounted for some differences in findings. We applied Bonferroni correction to adjust for multiple testing in our main analyses. However, this approach may have been too conservative in this exploratory study [59, 60]. Finally, as with all observational studies, unmeasured cofounding remains a limitation. We decided a priori to adjust only for sex and age at home visit. A thorough investigation of potential confounders and/or mediators would be required before any inference to causality could be made.

The two purposes of our study are the examination of the utility of newly emerging aging biomarkers, and the importance of growth and development in early life on ageing. Our findings suggest that in general these AgeAccel biomarkers do not capture the age-related effects of childhood growth. The second-generation AgeAccel biomarkers, particularly AgeAccelGrim, appear to be more sensitive to growth during puberty. The observed relationship between faster gains in weight during puberty, previously associated with cardiovascular risk, and AgeAccelGrim indicates that this period of growth in early life requires further investigation.

\section{Supplementary Information}

The online version contains supplementary material available at https://doi. org/10.1186/s13148-021-01138-x.

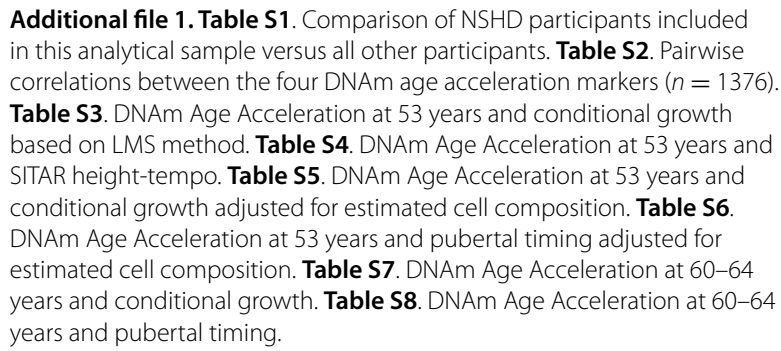

Additional file 1. Table S1. Comparison of NSHD participants included in this analytical sample versus all other participants. Table S2. Pairwise correlations between the four DNAm age acceleration markers $(n=1376)$. Table S3. DNAm Age Acceleration at 53 years and conditional growth based on LMS method. Table S4. DNAm Age Acceleration at 53 years and SITAR height-tempo. Table S5. DNAm Age Acceleration at 53 years and conditional growth adjusted for estimated cell composition. Table S6. DNAm Age Acceleration at 53 years and pubertal timing adjusted for estimated cell composition. Table S7. DNAm Age Acceleration at 60-64 years and conditional growth. Table S8. DNAm Age Acceleration at 60-64 years and pubertal timing.

\section{Acknowledgements}

We thank NSHD and NCDS study members for their lifelong participation and past and present members of the study teams, including Professor Alissa Goodman, Professor Ken Ong and members of the MRC Epidemiology unit in Cambridge, who helped to collect and process the data.

\section{Authors' contributions}

JM and RH designed the study. JM conducted the statistical analysis and wrote the manuscript. JCF performed the DNA methylation quality control. GBP, DK, AW, JB and RH obtained funding for the study. All authors read and approved the final manuscript.

\section{Funding}

This work was supported by the Economic and Social Research Council/Biotechnology and Biological Sciences Research Council [ES/N000404/1]. The UK 
Medical Research Council provides core funding for the MRC National Survey of Health and Development [MC_UU_00019/1]. RH is Director of CLOSER which is funded by the Economic and Social Research Council (award reference: ES/K000357/1)

\section{Availability of data and materials}

Data used in this publication are available to bona fide researchers upon request to the NSHD Data Sharing Committee via a standard application procedure. Further details can be found at http://www.nshd.mrc.ac.uk/data https://doi.org/10.5522/NSHD/S201. Information about NCDS can be found at https://cls.ucl.ac.uk/cls-studies/1958-national-child-development-study/ and data can be accessed via https://ukdataservice.ac.uk/.

\section{Declarations}

\section{Ethics approval and consent to participate}

The NSHD data collection at age 53 years received multicentre research ethics committee approval, and informed consent was given by respondents to each set of questions and measures. Ethical approval for the NCDS biomedical survey was obtained from South East Multi-centre Research Ethics Committee (ref. 01/1/44).

\section{Consent for publication}

Not applicable.

\section{Competing interests}

None declared.

\begin{abstract}
Author details
${ }^{1}$ MRC Unit for Lifelong Health and Ageing at UCL, Faculty of Population Health, University College London, 1-19 Torrington Place, London WC1E 7HB, UK. ${ }^{2}$ Department of Twin Research and Genetic Epidemiology, King's College London, London, UK. ${ }^{3}$ Centre for Longitudinal Studies, UCL Social Research Institute, University College London, London, UK. ${ }^{4}$ CLOSER, UCL Institute of Education, University College London, London WC1H ONU, UK.
\end{abstract}

Received: 5 January 2021 Accepted: 20 July 2021

Published online: 09 August 2021

\section{References}

1. Crimmins EM, Beltrán-Sánchez H. Mortality and morbidity trends: is there compression of morbidity? J Gerontol Ser B. 2010;66B(1):75-86.

2. Ben-Shlomo Y, Cooper R, Kuh D. The last two decades of life course epidemiology, and its relevance for research on ageing. Int J Epidemiol. 2016;45(4):973-88.

3. Ferrucci L, Levine ME, Kuo P-L, Simonsick EM. Time and the metrics of aging. Circ Res. 2018;123(7):740-4.

4. Jylhävä J, Pedersen NL, Hägg S. Biological age predictors. EBioMedicine. 2017;21:29-36.

5. Jones MJ, Goodman SJ, Kobor MS. DNA methylation and healthy human aging. Aging Cell. 2015;14(6):924-32.

6. Bocklandt S, Lin W, Sehl ME, Sánchez FJ, Sinsheimer JS, Horvath S, et al. Epigenetic predictor of age. PLoS ONE. 2011;6(6):e14821.

7. Hannum G, Guinney J, Zhao L, Zhang L, Hughes G, Sadda S, et al. Genome-wide methylation profiles reveal quantitative views of human aging rates. Mol Cell. 2013;49(2):359-67.

8. Horvath S. DNA methylation age of human tissues and cell types. Genome Biol. 2013;14(10):3156.

9. Horvath S, Raj K. DNA methylation-based biomarkers and the epigenetic clock theory of ageing. Nat Rev Genet. 2018;19(6):371-87.

10. Levine ME, Lu AT, Quach A, Chen BH, Assimes TL, Bandinelli S, et al. An epigenetic biomarker of aging for lifespan and healthspan. Aging (Albany NY). 2018;10(4):573-91.

11. Lu AT, Quach A, Wilson J, Reiner A, Aviv A, Hou L, et al. DNA methylation GrimAge strongly predicts lifespan and healthspan. Aging (Albany NY). 2019;11(2):303-27.

12. Levine ME. Assessment of epigenetic clocks as biomarkers of aging in basic and population research. J Gerontol Ser A. 2020;75(3):463-5.
13. Marioni RE, Shah S, McRae AF, Chen BH, Colicino E, Harris SE, et al. DNA methylation age of blood predicts all-cause mortality in later life. Genome Biol. 2015;16(1):25.

14. Chen BH, Marioni RE, Colicino E, Peters MJ, Ward-Caviness CK, Tsai P-C, et al. DNA methylation-based measures of biological age: meta-analysis predicting time to death. Aging (Albany NY). 2016;8(9):1844-65.

15. Perna L, Zhang Y, Mons U, Holleczek B, Saum K-U, Brenner H. Epigenetic age acceleration predicts cancer, cardiovascular, and all-cause mortality in a German case cohort. Clin Epigenet. 2016;8(1):64.

16. Dugué $P-A$, Bassett JK, Joo JE, Baglietto L, Jung C-H, Wong EM, et al. Association of DNA methylation-based biological age with health risk factors and overall and cause-specific mortality. Am J Epidemiol. 2017:187(3):529-38.

17. Maddock J, Castillo-Fernandez J, Wong A, Cooper R, Richards M, Ong KK, et al. DNA methylation age and physical and cognitive ageing. J Gerontol Ser A. 2019;75(3):504-11.

18. Hanson M, Gluckman P. Early developmental conditioning of later health and disease: physiology or pathophysiology? Physiol Rev. 2014;94(4):1027-76.

19. Ong KK, Loos RJ. Rapid infancy weight gain and subsequent obesity: systematic reviews and hopeful suggestions. Acta Paediatr. 2006:95(8):904-8.

20. Jones A, Charakida M, Falaschetti E, Hingorani AD, Finer N, Masi S, et al. Adipose and height growth through childhood and blood pressure status in a large prospective cohort study. Hypertension. 2012;59(5):919-25.

21. Hardy R, Ghosh AK, Deanfield J, Kuh D, Hughes AD. Birthweight, childhood growth and left ventricular structure at age 60-64 years in a British birth cohort study. Int J Epidemiol. 2016;45(4):1091-102.

22. Kuh D, Hardy R, Butterworth S, Okell L, Richards M, Wadsworth M, et al. Developmental origins of midlife physical performance: evidence from a British birth cohort. Am J Epidemiol. 2006;164(2):110-21.

23. Kuh D, Hardy R, Butterworth S, Okell L, Wadsworth M, Cooper C, et al. Developmental origins of midlife grip strength: findings from a birth cohort study. J Gerontol Ser A. 2006;61(7):702-6.

24. Richards M, Hardy R, Kuh D, Wadsworth ME. Birthweight, postnatal growth and cognitive function in a national UK birth cohort. Int J Epidemiol. 2002;31(2):342-8.

25. Peters SA, Woodward M. Women's reproductive factors and incident cardiovascular disease in the UK Biobank. Heart. 2018:104(13):1069-75.

26. Chen X, Liu Y, Sun X, Yin Z, Li H, Liu X, et al. Age at menarche and risk of all-cause and cardiovascular mortality: a systematic review and doseresponse meta-analysis. Menopause. 2019;26(6):670-6.

27. Kuh D, Muthuri SG, Moore A, Cole TJ, Adams JE, Cooper C, et al. Pubertal timing and bone phenotype in early old age: findings from a British birth cohort study. Int J Epidemiol. 2016;45(4):1113-24.

28. Binder AM, Corvalan C, Mericq V, Pereira A, Santos JL, Horvath S, et al. Faster ticking rate of the epigenetic clock is associated with faster pubertal development in girls. Epigenetics. 2018;13(1):85-94.

29. Suarez A, Lahti J, Czamara D, Lahti-Pulkkinen M, Girchenko P, Andersson S, et al. The epigenetic clock and pubertal, neuroendocrine, psychiatric, and cognitive outcomes in adolescents. Clin Epigenet. 2018;10(1):96.

30. Simpkin AJ, Howe LD, Tilling K, Gaunt TR, Lyttleton O, McArdle WL, et al. The epigenetic clock and physical development during childhood and adolescence: Iongitudinal analysis from a UK birth cohort. Int J Epidemiol. 2017;46(2):549-58.

31. Adair LS, Fall CH, Osmond C, Stein AD, Martorell R, Ramirez-Zea M, et al. Associations of linear growth and relative weight gain during early life with adult health and human capital in countries of low and middle income: findings from five birth cohort studies. The Lancet. 2013;382(9891):525-34.

32. Wadsworth M, Kuh D, Richards M, Hardy R. Cohort profile: the 1946 national birth cohort (MRC National Survey of Health and Development). Int J Epidemiol. 2005:35(1):49-54.

33. Kuh D, Wong A, Shah I, Moore A, Popham M, Curran P, et al. The MRC National Survey of Health and Development reaches age 70: maintaining participation at older ages in a birth cohort study. Eur J Epidemiol. 2016;31(11):1135-47.

34. Stafford M, Black S, Shah I, Hardy R, Pierce M, Richards M, et al. Using a birth cohort to study ageing: representativeness and response rates 
in the National Survey of Health and Development. Eur J Ageing. 2013;10(2):145-57.

35. Wadsworth M, Mann S, Rodgers B, Kuh D, Hilder W, Yusuf E. Loss and representativeness in a 43 year follow up of a national birth cohort. J Epidemiol Community Health. 1992;46(3):300-4.

36. Wadsworth ME, Butterworth S, Hardy R, Kuh D, Richards M, Langenberg C, et al. The life course prospective design: an example of benefits and problems associated with study longevity. Soc Sci Med. 2003:57(11):2193-205.

37. Xu Z, Niu L, Li L, Taylor JA. ENmix: a novel background correction method for Illumina HumanMethylation450 BeadChip. Nucleic Acids Res. 2016;44(3):e20-e.

38. McEwen LM, Jones MJ, Lin DTS, Edgar RD, Husquin LT, Maclsaac JL, et al. Systematic evaluation of DNA methylation age estimation with common preprocessing methods and the Infinium MethylationEPIC BeadChip array. Clin Epigenet. 2018;10(1):1-9.

39. Fortin J-P, Triche TJ Jr, Hansen KD. Preprocessing, normalization and integration of the Illumina HumanMethylationEPIC array with minfi. Bioinformatics. 2017;33(4):558-60.

40. Hardy R, Kuh D, Whincup PH, Wadsworth ME. Age at puberty and adult blood pressure and body size in a British birth cohort study. J Hypertens. 2006;24(1):59-66.

41. Cooper R, Blell M, Hardy R, Black S, Pollard T, Wadsworth M, et al. Validity of age at menarche self-reported in adulthood. J Epidemiol Community Health. 2006:60(11):993-7.

42. Cole TJ, Kuh D, Johnson W, Ward K, Howe LD, Adams JE, et al. Using Super-Imposition by Translation And Rotation (SITAR) to relate pubertal growth to bone health in later life: the Medical Research Council (MRC) National Survey of Health and Development. Int J Epidemiol. 2016;45(4):1125-34

43. De Stavola BL, Nitsch D, dos Santos SI, McCormack V, Hardy R, Mann $V$, et al. Statistical issues in life course epidemiology. Am J Epidemiol. 2005;163(1):84-96.

44. Tu Y-K, Tilling K, Sterne JA, Gilthorpe MS. A critical evaluation of statistical approaches to examining the role of growth trajectories in the developmental origins of health and disease. Int J Epidemiol. 2013:42(5):1327-39.

45. Wills AK, Strand BH, Glavin K, Silverwood RJ, Hovengen R. Regression models for linking patterns of growth to a later outcome: infant growth and childhood overweight. BMC Med Res Methodol. 2016;16(41):366.

46. Johnson W. Analytical strategies in human growth research. Am J Hum Biol. 2015;27(1):69-83.

47. Cole T. Using the LMS method to measure Skewness in the NCHS and Dutch National height standards. Ann Hum Biol. 1989;16(5):407-19.

48. Hardy R, Wills AK, Wong A, Elks CE, Wareham NJ, Loos RJ, et al. Life course variations in the associations between FTO and MC4R gene variants and body size. Hum Mol Genet. 2010;19(3):545-52.
49. Silverwood RJ, Leon DA, De Stavola BL. Long-term trends in BMI: are contemporary childhood BMl growth references appropriate when looking at historical datasets? Longitud Life Course Stud. 2009;1 (1):27-44.

50. Power C, Elliott J. Cohort profile: 1958 British birth cohort (National Child Development Study). Int J Epidemiol. 2006;35(1):34-41.

51. Simpkin AJ, Hemani G, Suderman M, Gaunt TR, Lyttleton O, Mcardle WL, et al. Prenatal and early life influences on epigenetic age in children: a study of mother-offspring pairs from two cohort studies. Hum Mol Genet. 2015;25(1):191-201.

52. Ryan J, Wrigglesworth J, Loong J, Fransquet PD, Woods RL. A systematic review and meta-analysis of environmental, lifestyle and health factors associated with DNA methylation age. J Gerontol Ser A. 2019;75(3):481-94.

53. Hardy R, Maddock J, Ghosh AK, Hughes AD, Kuh D. The relationship between pubertal timing and markers of vascular and cardiac structure and function in men and women aged 60-64 years. Sci Rep. 2019;9:11037.

54. Pérez-de-Heredia F, Gómez-Martínez S, Díaz L-E, Veses AM, Nova E, Wärnberg J, et al. Influence of sex, age, pubertal maturation and body mass index on circulating white blood cell counts in healthy European adolescents - the HELENA study. Eur J Pediatr. 2015;174(8):999-1014.

55. El Khoury LY, Gorrie-Stone T, Smart M, Hughes A, Bao Y, Andrayas A, et al. Systematic underestimation of the epigenetic clock and age acceleration in older subjects. Genome Biol. 2019;20(1):1-10.

56. Shireby GL, Davies JP, Francis PT, Burrage J, Walker EM, Neilson GW, et al. Recalibrating the epigenetic clock: implications for assessing biological age in the human cortex. Brain. 2020;143(12):3763-75.

57. Whitley E, Martin RM, Smith GD, Holly JMP, Gunnell D. The association of childhood height, leg length and other measures of skeletal growth with adult cardiovascular disease: the Boyd-Orr cohort. J Epidemiol Community Health. 2012;66(1):18-23.

58. Munafò MR, Tilling K, Taylor AE, Evans DM, Davey SG. Collider scope: when selection bias can substantially influence observed associations. Int J Epidemiol. 2018;47(1):226-35.

59. Perneger TV. What's wrong with Bonferroni adjustments. BMJ. 1998;316(7139):1236-8

60. Rothman KJ. No adjustments are needed for multiple comparisons. Epidemiology. 1990;15:43-6.

\section{Publisher's Note}

Springer Nature remains neutral with regard to jurisdictional claims in published maps and institutional affiliations.
Ready to submit your research? Choose BMC and benefit from:

- fast, convenient online submission

- thorough peer review by experienced researchers in your field

- rapid publication on acceptance

- support for research data, including large and complex data types

- gold Open Access which fosters wider collaboration and increased citations

- maximum visibility for your research: over 100M website views per year

At BMC, research is always in progress.

Learn more biomedcentral.com/submissions 